EPJ manuscript No.

(will be inserted by the editor)

\title{
A theoretical view on bound antikaon-nuclear states
}

\author{
A. Ramos ${ }^{1}$, V. K. Magas ${ }^{1}$, E. Oset ${ }^{2}$, and H. Toki ${ }^{3}$ \\ 1 Departament d'Estructura i Constituents de la Matèria, Universitat de Barcelona, Diagonal 647, 08028 Barcelona, Spain \\ 2 Departamento de Física Teórica and IFIC Centro Mixto Universidad de Valencia-CSIC, Institutos de Investigación de Paterna \\ Apdo. correos 22085, 46071, Valencia, Spain \\ 3 Research Center for Nuclear Physics, Osaka University, Ibaraki, Osaka 567-0047, Japan
}

Received: date / Revised version: date

\begin{abstract}
We present an overview of the latest theoretical studies on the antikaon properties in the nuclear medium, in connection with the recent experimental claims of very deeply bound antikaon-nuclear states. We argue that proper many-body formulations using modern realistic antikaon-nucleon interactions are not able to generate such systems. Instead, a simple two-nucleon antikaon absorption mechanism where the remaining nucleus acts as spectator explains the peak in the semi-inclusive proton momentum spectrum, observed on a ${ }^{4} \mathrm{He}$ target at KEK (but later not confirmed in an inclusive experiment) and on a ${ }^{6} \mathrm{Li}$ target at FINUDA. This signal is clearly seen in another FINUDA experiment measuring the invariant mass of $\Lambda$-proton pairs after two-nucleon kaon absorption. We show that another peak of this experiment, seen at lower invariant masses and interpreted as a bound $K^{-} p p$ state, is simply generated by the same two-nucleon absorption mechanism followed by final-state interactions of the produced particles with the residual nucleus. Our conclusion is that all the experimental claims for the formation of very deeply bound antikaonic nuclear systems receive an alternative explanation in terms of conventional nuclear processes.
\end{abstract}

PACS. $25.80 . \mathrm{Nv}-13.75 . \mathrm{Jz}-21.45 .+\mathrm{v}-21.80 .+\mathrm{a}$

\section{Introduction}

Over the last years, the theoretical study of the interactions of antikaons with nuclei has received a lot of attention due to the important implications on the feasible realization of interesting physics phenomena. The possible appearance of a condensate of antikaons in neutron stars was postulated after examining the sizable attractive properties of the chiral $\bar{K} N$ interaction at tree level [1]. Phenomenological fits to kaonic atoms fed the idea because a solution were antikaons would feel strongly attractive potentials of the order of $-200 \mathrm{MeV}$ at the center of the nucleus 2 was preferred. However, a more satisfactory theoretical understanding of the size of the antikaon optical potential demands it to be linked to the elementary $\bar{K} N$ scattering amplitude which is dominated by the presence of a resonance, the $\Lambda(1405)$, located only $27 \mathrm{MeV}$ below threshold. This makes the problem to be a highly non-perturbative one. In recent years, the scattering of $\bar{K}$ mesons with nucleons has been treated within the context of chiral unitary methods $3,4,5,6,7,8,9,10$. The explicit incorporation of medium effects, such as Pauli blocking, were shown to be important [1] and it was soon realized that the influence of the resonance demanded the in-medium amplitudes to be evaluated self-consistently [12. The resulting antikaon optical potentials were quite shallower than the phenomenological one, with depths be- tween -70 and $-40 \mathrm{MeV}$ 13, 14, 15, but gave an acceptable description of the kaonic atom data [16,17.

More recently, variational calculations of few body systems using a simplified phenomenological $\bar{K} N$ interaction predicted extremely deep kaonic states in ${ }^{3} \mathrm{He}$ and ${ }^{4} \mathrm{He}$, reaching densities of up to ten times that of normal nuclear matter [18,19,20]. Motivated by this finding, experiment KEK-E471 used the ${ }^{4} \mathrm{He}$ (stopped $\left.K^{-}, p\right)$ reaction and reported 21] a structure in the proton momentum spectrum, which was denoted as the tribaryon $S^{0}(3115)$ with strangeness $S=-1$. If interpreted as a $\left(K^{-} p n n\right)$ bound state, it would have a binding energy of $194 \mathrm{MeV}$. However, in a recent work 22] strong criticisms to the theoretical claims of Refs. 18, 19,20 have been put forward and, in addition, a reinterpretation of the KEK proton peak has been given in terms of two nucleon absorption processes, $K^{-} p n \rightarrow \Sigma^{-} p, K^{-} p p \rightarrow \Sigma^{0}(\Lambda) p$, where the rest of the nucleus acts as a spectator. We note that deficiencies in the efficiency corrections were revealed in the last HYP06 conference, and the new reanalyzed KEK proton momentum spectrum does not show evidence of this peak [23, although the conditions of the analysis (cuts and coincidences with other charged particles) were not the same as in the previous one. In contrast, a FINUDA proton momentum spectrum from $K^{-}$absorption on ${ }^{6} \mathrm{Li}$ 24] does show a peak, which is interpreted with the twonucleon absorption mechanism advocated in Ref. [22. 
Another experiment of the FINUDA collaboration has measured the invariant mass distribution of $\Lambda p$ pairs [25. The spectrum shows a narrow peak at $2340 \mathrm{MeV}$, which corresponds to the same signal seen in the proton momentum spectrum, namely $K^{-}$absorption by a two-nucleon pair leaving the daughter nucleus in its ground state. Another wider peak is also seen at around $2255 \mathrm{MeV}$, which is interpreted in Ref. 25] as being a $K^{-} p p$ bound state with $B_{K^{-} p p}=115_{-5}^{+6}(\text { stat })_{-3}^{+2}$ (syst) $\mathrm{MeV}$ and having a width of $\Gamma=67_{-11}^{+14}$ (stat) $)_{-3}^{+2}$ (syst) $\mathrm{MeV}$. In a recent work [26] we showed that this peak is generated from the interactions of the $\Lambda$ and nucleon, produced after $K^{-}$absorption, with the residual nucleus. We here summarize the present status of the theoretical studies on the antikaon properties in the nuclear medium, in connection with the recent experimental claims of very deeply bound antikaon-nuclear states.

\section{Kaon-nucleon interaction and kaon-nucleus optical potential}

The existence of deeply bound kaonic states is linked to the properties of the antikaon-nucleon $(\bar{K} N)$ interaction and its modification due to the nuclear medium. In particular, the understanding of the $\Lambda(1405)$, which is $27 \mathrm{MeV}$ below the $\bar{K} N$ threshold and decays exclusively into $\pi \Sigma$ states, is essential in order to study the fate of the kaons in the nucleus. The properties of the $\Lambda(1405)$ were nicely reproduced long ago from the solution of the Schrödinger equation in two $(\bar{K} N, \pi \Sigma)$ coupled channels [27]. The model also reproduced a repulsive $K^{-} p$ amplitude at threshold, a feature which is well known both from extrapolation of scattering data [28, and from the measurement of kaonic hidrogen at KEK [29] and DA $\Phi$ NE [30].

All the features discussed above are worked out more systematically in the $\mathrm{SU}(3)_{f}$ chiral unitary models $[3,4,5$, 6, 7, 8, 9, 10, where one uses input from chiral Lagrangians and, in addition to the $\bar{K} N, \pi \Sigma$ channels, one also has other channels from the combination of the octet of pseudoscalar mesons with the octet of stable baryons. The chiral models generate the $\Lambda(1405)$ and reproduce various $K^{-} p$ reactions. In addition, the $\Lambda(1405)$ is revealed as being the superposition of two poles of the scattering matrix, one appearing around $1385 \mathrm{MeV}$, with a width of $140 \mathrm{MeV}$, and coupling mostly to $\pi \Sigma$, the other appearing around $1420 \mathrm{MeV}$, with a $30 \mathrm{MeV}$ width and coupling mostly to $\bar{K} N$. This is corroborated by the analysis of the $K^{-} p \rightarrow \pi^{0} \pi^{0} \Sigma^{0}$ data [31] using the chiral model amplitudes 32 .

The procedure to obtain the meson-baryon amplitude in the chiral unitary models is extremely simple. From the lowest order chiral lagrangian for the meson baryon interaction,

$L_{1}^{(B)}=\left\langle\bar{B} i \gamma^{\mu} \frac{1}{4 f^{2}}\left[\left(\Phi \partial_{\mu} \Phi-\partial_{\mu} \Phi \Phi\right) B-B\left(\Phi \partial_{\mu} \Phi-\partial_{\mu} \Phi \Phi\right)\right]\right\rangle$

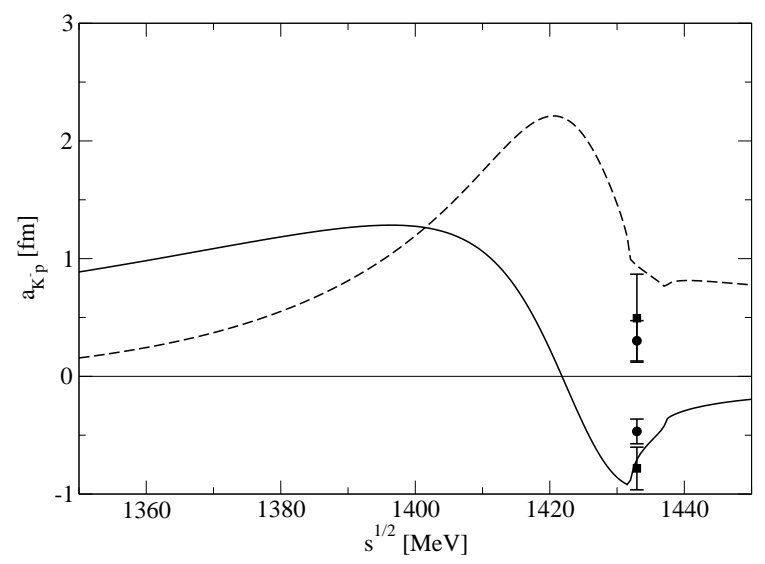

Fig. 1. Real (solid) and imaginary (dotted) parts of the $K^{-} p$ scattering amplitude, as functions of the $\bar{K} N$ center of mass energy $\sqrt{s}$. The real and imaginary parts of the scattering length measured at KEK 29] (squares) and DA $\Phi$ NE [30] (dots) are also displayed.

where $\Phi$ and $B$ are the ordinary $\mathrm{SU}(3)$ matrices of the meson and baryon fields respectively, one deduces the swave kernel of the Bethe-Salpeter equation (potential V):

$V_{i j}=-C_{i j} \frac{1}{4 f^{2}}\left(2 \sqrt{s}-M_{i}-M_{j}\right) \sqrt{\frac{M_{i}+E_{i}}{2 M_{i}}} \sqrt{\frac{M_{j}+E_{j}}{2 M_{j}}}$,

where $M_{i}$ and $E_{i}$ denote the mass and energy of the baryon in channel $i$. The coefficients of the symmetric matrix $C_{i j}$ are given in [4. The scattering amplitude is then obtained by solving the coupled-channel Bethe-Salpeter equation,

$$
T=[1-V G]^{-1} V,
$$

using on-shell amplitudes. The loop function $G$ must be regularized by means of either a cut-off or by a subtraction method in dimensional regularization. The real and imaginary parts of the $K^{-} p$ scattering length,

$$
a_{K^{-} p}=-\frac{1}{4 \pi} \frac{M_{p}}{\sqrt{s}} T_{K^{-} p \rightarrow K^{-} p},
$$

obtained from the lowest order chiral model of Ref. 4, but using the dimensional regularization scheme described in [8], are displayed in Fig. 1] Note that the real part is negative (repulsive) at threshold and agrees with the KEK data 29 but not with the recent DEAR determination [30], which is about $30 \%$ smaller. A recent model, including also the next to leading order terms in the chiral kernel [9] achieves a better agreement with the real part of the DEAR scattering length, but still misses the imaginary part by about half the size.

One needs next to implement the corrections to the $\bar{K} N$ amplitude in the medium. Pauli blocking acting on the intermediate loop nucleons forces them to be placed above de Fermi momentum, which costs more energy and induces a repulsive effect on the resonance. Correspondingly, the $K^{-} p$ amplitude at threshold changes from being repulsive to being attractive, providing in this way a 
source of attraction for the $\bar{K}$ self-energy or optical potential. One should not stop here since the antikaons in the intermediate states feel this attractive potential. It then costs less energy to excite them, which partly compensates the repulsive Pauli blocking effect. As a consequence, the resonance moves back to lower energies and the new kaon self-energy will not be as attractive. This self-consistency procedure was done for the first time in Ref. 12. It was found that the attraction felt by the kaons was drastically reduced. Subsequent calculations 14,15, including those which consider also the renormalization of the intermediate pions in the $\pi \Sigma$ channel [13] were performed and the strength of the potential was shown to be attractive and of the order of 40-70 MeV at nuclear matter density.

Indeed, from the self-consistent $\bar{K} N$ amplitudes obtained following the model of Ref. 13, improved by including the p-wave components of the kaon self-energy renormalized by the effect of short-range correlations [33, one obtains the antikaon optical potential displayed in Fig. 2 as a function of the antikaon momentum at normal nuclear density, $\rho=0.16 \mathrm{fm}^{-3}$. The real part of the optical potential, evaluated at the corresponding quasiparticle energy, $\varepsilon_{K}=k_{K}^{2} /\left(2 m_{K}\right)+\operatorname{Re} U_{K}$, is attractive and around $-50 \mathrm{MeV}$ at zero momentum. The attraction is somewhat larger if the dressing of pions in the intermediate loops is ignored, as done in most calculations. It is important to notice that the self-consistent procedure, including also the p-wave excitation of $\Lambda$-hole and $\Sigma$-hole components in the antikaon self-energy, automatically incorporates the absorption of the antikaons by two nucleons, namely $K^{-} N N \rightarrow N \Lambda, N \Sigma$, in the imaginary part of the optical potential. The dressing of the pion brings an additional source of absorption strength through this processes but also through new channels (via the $\Delta$-hole pionic excitations), $K^{-} N N \rightarrow \pi N \Lambda, \pi N \Sigma$, which turn out to be very relevant in the low momentum region of the kaon optical potential.

This potential, evaluated at $k_{K}=0 \mathrm{MeV}$ and $\varepsilon_{K}=$ $m_{K}$, provides a good reproduction of the kaonic atom data in light and medium heavy nuclei [16, with small diversions that were quantified in a best fit analysis [17. We can then conclude that the $\mathrm{SU}(3)$ chiral unitary model with the inclusion of all the medium corrections on the baryons and the mesons is supported from the kaonic atom data.

With this behavior for the $\bar{K} N$ dynamics in nuclei having been established, the claim given in 18, 19 on the possible existence of narrow deeply bound states in light nuclei, predicting $A=3 I=0$ and $I=1$ states with binding energies of around $100 \mathrm{MeV}$, was surprising. The situation became more puzzling when an experiment searching for deeply bound $K^{-}$states using the reaction ${ }^{4} \mathrm{He}\left(\right.$ stopped $\left.K^{-}, p\right)[21$ found a signal for a $I=1$ strange tri-baryon, $S^{0}(3115)$, since its interpretation as a bound state would imply a binding energy around $200 \mathrm{MeV}$. Subsequent corrections including relativistic effects [20], supplied the necessary amount of attraction, yielding a $\bar{K}$ nucleus potential strength of $-600 \mathrm{MeV}$ and nuclear densities at the center of the nucleus of about 9 times nuclear matter saturation density. A closer look at the model in-

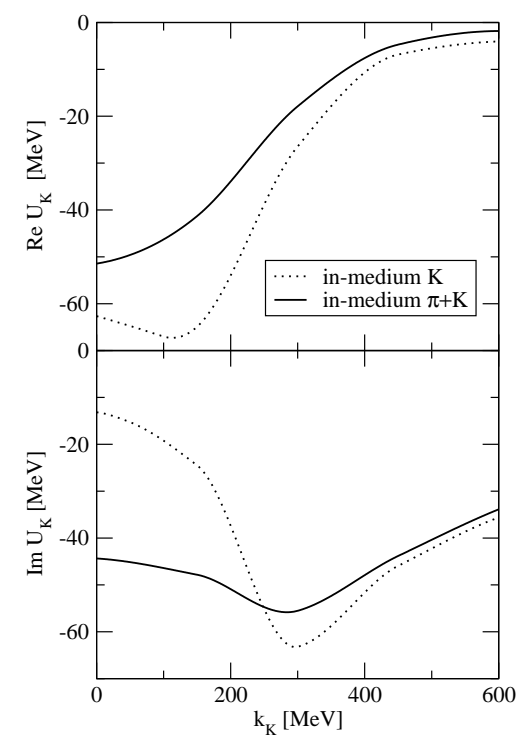

Fig. 2. Real (top) and imaginary (bottom) part of the $K^{-}$ optical potential as a function of momentum obtained from two approximations: in-medium $K$ (dotted lines): only antikaons are (self-consistently) dressed and in-medium $\pi+K$ (solid lines): pions and antikaons are dressed.

teraction and the many-body approximations employed in these works reveals serious deficiencies that were pointed out in Ref. 22. See also the criticisms put forward in Ref. 34. Among those: 1) The theoretical potential in [18, 19, 20, eliminates the direct coupling of $\pi \Sigma \rightarrow \pi \Sigma$ and $\pi \Lambda \rightarrow \pi \Lambda$ in contradiction with the results of chiral theory that establishes large couplings for them 4. 2) Selfconsistency, shown to be essential in regulating the size of attraction felt by the antikaons, was not implemented. 3) Finally, short range correlations, which would prevent reaching the high densities found, were not incorporated realistically.

An important turnover in the understanding of deeply bound kaonic systems has been given by recent few body calculations applied to the $K^{-} p p$ system. Three-body coupled channel Faddeev equations are solved in Ref. 35, using separable two-body interactions fitted to scattering data. A $I=1 / 2$ three-body $\bar{K}(N N)_{I=1}$ state was found with a binding energy in the range $B \sim 50-70$ $\mathrm{MeV}$ and a width $\Gamma \sim 95-110 \mathrm{MeV}$. The recent variational calculations of Ref. 36 implement, as a major change with respect the previous works $[18,19,20$, realistic short-range NN correlations, which prevent from reaching the high central densities obtained before. Although still preliminary, the first results point at a binding energy for the $K^{-} p p$ system of around $50 \mathrm{MeV}$ and a total width for this state larger than $100 \mathrm{MeV}$, the absorption $K^{-} N N \rightarrow Y N$ channel, not considered in 35], contributing by about $20 \%$. 
A. Ramos et al.: A theoretical view on bound antikaon-nuclear states

\section{Proton momentum spectrum}

Convinced by the outcome of realistic many-body calculations, both in nuclear matter and in finite nuclei, that the peak in the proton momentum spectrum found at KEK 21] could not correspond to a deeply bound $K^{-}$ state, the authors of Ref. [22] searched for other explanations and found a natural mechanism that passed all tests. The peak is due to the two-body absorption mechanism, $K^{-} N N \rightarrow \Sigma p$, in ${ }^{4}$ He leaving the other nucleons as spectators. The candidate reaction to produce a peak is $K^{-4} \mathrm{He} \rightarrow \Sigma^{-} p d$, which has a production rate of $1.6 \%$ 37. This is compatible with demanding that $1 \%$ of the strength proceeds through the $d$ acting as a spectator, as required by the two-body absorption mechanism, which is the formation branching ratio per stopped $K^{-}$for the peak seen in Ref. 21] just below $500 \mathrm{MeV} / \mathrm{c}$. The two nucleon absorption mechanism produces a well defined narrow peak at $p_{p} \sim 480 \mathrm{MeV} / \mathrm{c}$, as in the experiment, if the Fermi motion of the spectator $d$ is ignored, which is equivalent in this mechanism to ignoring the corresponding recoil momentum. These effects have been shown 38 to produce a broadening of the proton peak of $\sim 70 \mathrm{MeV}$ for $K^{-}$ absorption in ${ }^{4} \mathrm{He}$ and of $\sim 20 \mathrm{MeV}$ if the absorption takes place in ${ }^{6} \mathrm{Li}$. Actually, the peaks can be narrower and/or enhanced if some specific kinematical cuts are applied. For instance, a secondary charged particle is also detected, together with the proton, in the KEK experiment and the peak becomes more prominent when this secondary particle is a $\pi^{-}$. We note that the KEK group reported recently 23 some deficiencies in correcting the proton efficiencies of their previous experiment, and the new proton spectrum from the reaction ${ }^{4} \mathrm{He}$ (stopped $\left.K^{-}, p\right)$ no longer shows a peak. It remains to be seen if the peak would reappear if the same semi-inclusiveness conditions as in the earlier experiment were applied. What is clear is that recoil-broadening effects of $70 \mathrm{MeV}$ plus formation rates of $\sim 1 \%$ make the observation of this peak hard, unless specific kinematical and particle selection cuts are applied. This is precisely what was done in the proton momentum spectrum reported by the FINUDA collaboration from $K^{-}$capture on ${ }^{6} \mathrm{Li} 24$, where the peak observed at around $500 \mathrm{MeV} / \mathrm{c}$ was correlated with a $\pi^{-}$coming from $\Sigma^{-}$decay in flight, selected by choosing pion momenta larger than $275 \mathrm{MeV} / \mathrm{c}$. It was also shown there that most of the events fulfilled the back-to-back condition $\cos \theta_{\pi^{-}}<-0.8$, confirming the origin of the proton peak as coming from the two-nucleon $K^{-}$absorption mechanism. We note that this interpretation demanded the peak corresponding to the reaction $K^{-} N N \rightarrow \Lambda p$ be seen as well, which was indeed the case in the FINUDA experiment 24] where a feeble signal can be observed at about $580 \mathrm{MeV} / \mathrm{c}$.

The signals in the proton spectrum should appear when a proton as well as a $\Sigma$ or a $\Lambda$ would be emitted back to back after two-nucleon $K^{-}$absorption and a residual nuclear system remains as a spectator and stays nearly in its ground state. This last requirement becomes more difficult in heavier nuclei since the distortion of the $\Lambda$ or $p$ particle in their way out through the nucleus leads un- avoidably to nuclear excitations. As a consequence of this, one expects the signals to fade gradually for heavier nuclei, which would explain the smoothness of the proton spectrum from absorption on ${ }^{12} \mathrm{C}$, also measured in [24].

\section{The $\Lambda p$ invariant mass spectrum}

Having thus reinterpreted the alleged deeply bound kaonic state, reported from the proton spectrum experiment E471 at KEK, as simply coming from a two-nucleon $K^{-}$absorption mechanism, a reanalysis of another FINUDA experiment, claiming to have formed a $K^{-} p p$ state bound by $115 \mathrm{MeV}$ seen in a $\Lambda p$ invariant mass spectrum [25, is an absolute necessity. This was done in Ref. [26] and, in this section, we summarize the main ideas and results of that work, implementing some minor improvements.

The FINUDA collaboration in 25] looked for $\Lambda p$ events back to back following the $K^{-} p p$ absorption in ${ }^{6} \mathrm{Li},{ }^{7} \mathrm{Li}$ and ${ }^{12} \mathrm{C}$ nuclei. We have developed a simulation code for the reaction $K^{-} A \rightarrow \Lambda p A^{\prime}$ with stopped kaons, which accounts for the final state interaction of the produced $\Lambda$ and $p$ with the residual nuclear state. Once this is done, the $\Lambda p$ events are collected considering the cuts applied in the experiment: one selecting $p_{\Lambda}>300 \mathrm{MeV} / \mathrm{c}$, to eliminate events from $K^{-} p \rightarrow \Lambda \pi$, and another one imposing $\cos \Theta_{\boldsymbol{p}_{\Lambda} \boldsymbol{p}_{p}}<-0.8$, to filter $\Lambda p$ pairs going back to back.

More specifically, the reaction $\left(K^{-}\right)_{\text {stopped }} A \rightarrow \Lambda p A^{\prime}$ proceeds by capturing a slow $K^{-}$in a high atomic orbit of the nucleus, which later cascades down till the $K^{-}$reaches a low lying orbit, from where it is finally absorbed. We assume that the absorption of the $K^{-}$takes place from the lowest level where the energy shift for atoms has been measured, or, if it is not measured, from the level where the calculated shift [16] falls within the measurable range.

The width for $K^{-}$absorption from $p N$ pairs in a nucleus with mass number $A$ is given, in Local Density Approximation (LDA) by

$$
\Gamma_{A} \propto \int d^{3} \boldsymbol{r}\left|\Psi_{K^{-}}(\boldsymbol{r})\right|^{2} \int \frac{d^{3} \boldsymbol{p}_{1}}{(2 \pi)^{3}} \int \frac{d^{3} \boldsymbol{p}_{2}}{(2 \pi)^{3}} \Gamma_{m}\left(\boldsymbol{p}_{1}, \boldsymbol{p}_{2}, \boldsymbol{p}_{K}, \boldsymbol{r}\right),
$$

where $\left|\Psi_{K^{-}}(\boldsymbol{r})\right|^{2}$ is the probability of finding the $K^{-}$in the nucleus, $\left|\boldsymbol{p}_{1}\right|,\left|\boldsymbol{p}_{2}\right|<k_{F}(r)$ with $k_{F}(r)=\left(3 \pi^{2} \rho(r) / 2\right)^{1 / 3}$ being the local Fermi momentum and $\Gamma_{m}\left(\boldsymbol{p}_{1}, \boldsymbol{p}_{2}, \boldsymbol{p}_{K}, \boldsymbol{r}\right)$ is the in-medium decay width for the $K^{-} p N \rightarrow \Lambda N$ process. The structure of the integrals determining $\Gamma_{m}$,

$$
\Gamma_{m} \propto \int d^{3} \boldsymbol{p}_{\Lambda} d^{3} \boldsymbol{p}_{N} \ldots K\left(\boldsymbol{p}_{\Lambda}, \boldsymbol{r}\right) K\left(\boldsymbol{p}_{N}, \boldsymbol{r}\right),
$$

allows us to follow the propagation of the produced nucleon and $\Lambda$ through the nucleus after $K^{-}$absorption via the kernel $K(\boldsymbol{p}, \boldsymbol{r})$. The former two equations describe the process in which a kaon at rest is absorbed by two nucleons ( $p p$ or $p n$ ) within the local Fermi sea emitting a nucleon and a $\Lambda$. The primary nucleon $(\Lambda)$ is allowed to re-scatter with nucleons in the nucleus according to a probability per unit length given by $\sigma_{N(\Lambda)} \rho(r)$, where $\sigma_{N(\Lambda)}$ is the 


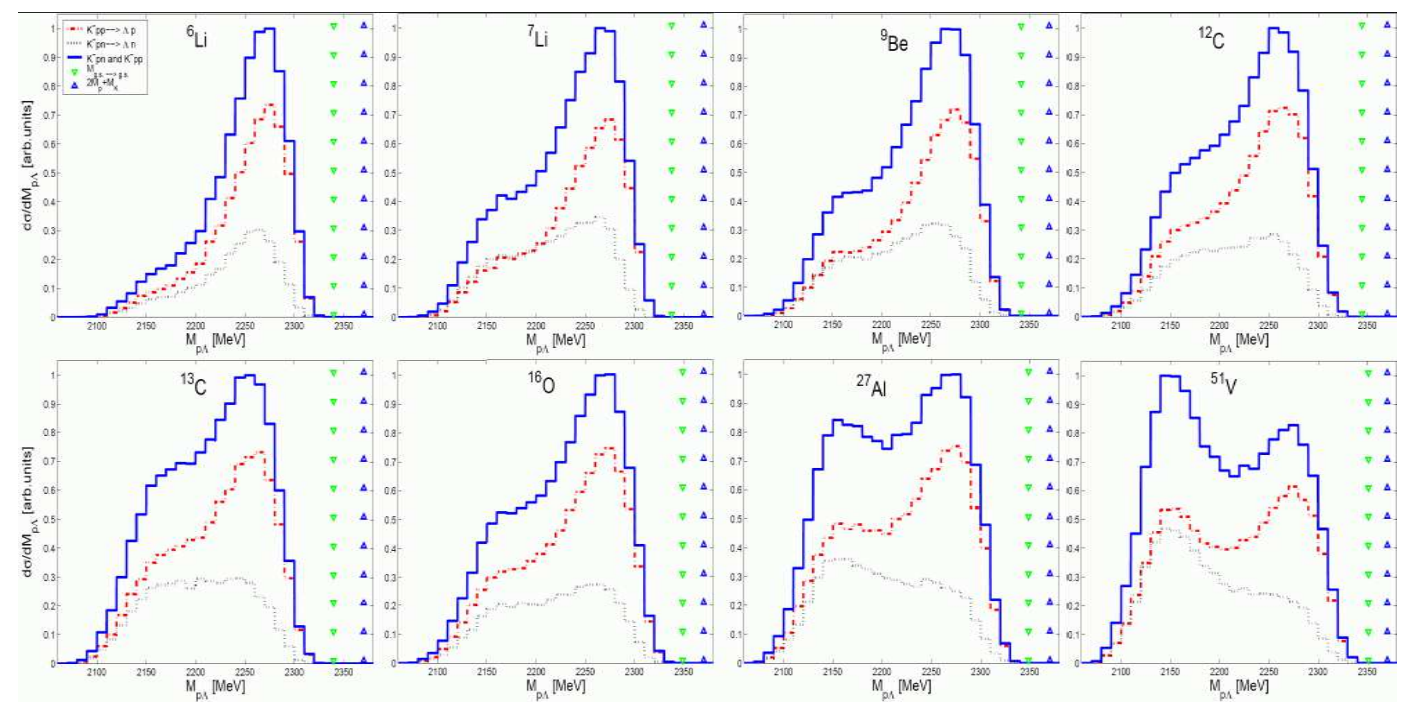

Fig. 3. $\Lambda p$ invariant mass distribution after $K^{-}$absorption in several nuclei imposing $P_{\Lambda}>300 \mathrm{MeV} / \mathrm{c}$ and $\cos \Theta_{\boldsymbol{p}_{\Lambda} \boldsymbol{p}_{p}}<-0.8$.

experimental $N N(\Lambda N)$ cross section at the corresponding energy, while in [26] a simpler parameterization for the $\Lambda$, of the type $\sigma_{\Lambda}=2 \sigma_{N} / 3$ was employed. The angular distribution of the re-scattered particles are also generated according to experimental differential cross-sections. We note that particles move under the influence of a mean field potential, of Thomas-Fermi type. After several possible collisions, one or more nucleons and a $\Lambda$ emerge from the nucleus and the invariant mass of all possible $\Lambda p$ pairs, as well as their relative angle, are evaluated for each Monte Carlo event. See Ref. 26] for more details.

Absorption of a $K^{-}$from a nucleus leaving the final daughter nucleus in its ground state gives rise to a narrow peak in the $\Lambda p$ invariant mass distribution, as it is observed in the spectrum of 25. We note that our local density formalism, in which the hole levels in the Fermi sea form a continuum of states, cannot handle properly transitions to discrete states of the daughter nucleus, in particular to the ground state. For this reason, we will remove in our calculations those events in which the $p$ and $\Lambda$ produced after $K^{-}$absorption leave the nucleus without having suffered a secondary collision. However, due to the small overlap between the two-hole initial state after $K^{-}$ absorption and the residual $(A-2)$ ground state of the daughter nucleus, as well as to the limited survival probability for both the $p$ and the $\Lambda$ crossing the nucleus without any collision, this strength represents only a moderate fraction, estimated to be smaller than $15 \%$ in ${ }^{7} \mathrm{Li}[26$. This means in practice that the excitation of the nucleus will require the secondary collision of the $p$ or $\Lambda$ after the $K^{-} p p$ absorption process, similarly as to what happens in $\left(p, p^{\prime}\right)$ collisions, where the strength of the cross section to elastic or bound excited states is very small compared to that of nuclear breakup producing the quasi-elastic peak.

Our invariant mass spectra requiring at least a secondary collision of the $p(n)$ or $\Lambda$ after the $K^{-} p p(n p)$ absorption process are shown in Fig. 3 for several nuclei, where we have applied the same cuts as in the experi- ment, namely $P_{\Lambda}>300 \mathrm{MeV} / \mathrm{c}$ (to eliminate events from $K^{-} p \rightarrow \Lambda \pi$ ) and $\cos \Theta_{\boldsymbol{p}_{\Lambda} \boldsymbol{p}_{p}}<-0.8$ (to filter $\Lambda p$ pairs going back-to-back). Actually, the calculated angular distribution shown in Ref. 26 demonstrates that, even after collisions, a sizable fraction of the events appear at the back-to-back kinematics. These events generate the main bump at $2260-2270 \mathrm{MeV}$ in all the $\Lambda p$ invariant mass spectra shown in Fig. 3, about the same position as the main peak shown in the inset of Fig. 3 of 25. We note that, since one measures the $\Lambda p$ invariant mass, the main contribution comes from $K^{-} p p \rightarrow \Lambda p$ absorption (dot-dashed lines), although the contribution from the $K^{-} p n \rightarrow \Lambda n$ reaction followed by $n p \rightarrow p n$ (dotted line) is non-negligible. It is interesting to observe that the width of the distribution gets broader with the size of the nucleus, while the peak remains in the same location, consistently to what one expects for the behavior of a quasi-elastic peak. Let us point out in this context that the work of [39] shows that the possible interpretation of the FINUDA peak as a bound state of the $K^{-}$with the nucleus, not as a $K^{-} p p$ bound state, would unavoidably lead to peaks at different energies for different nuclei. We finally observe that the spectra of heavy nuclei develop a secondary peak at lower invariant masses due to the larger amount of re-scattering processes. It is slightly more pronounced than that shown in our earlier work [26], due to the use here of a more accurate $\Lambda N$ scattering cross section.

Finally, we compare our results with those presented in the inset of Fig. 3 in Ref. 25, using the three lighter targets in the same proportion as in the experiment $(51 \%$ ${ }^{12} \mathrm{C}, 35 \%{ }^{6} \mathrm{Li}$ and $\left.14 \%{ }^{7} \mathrm{Li}\right)$. We note that the averaged histogram is dominated by the ${ }^{12} \mathrm{C}$ component of the mixture, due mostly to the larger overlap with the kaon wave function. As we see, our calculations are in excellent agreement with the measured spectrum in this range. 


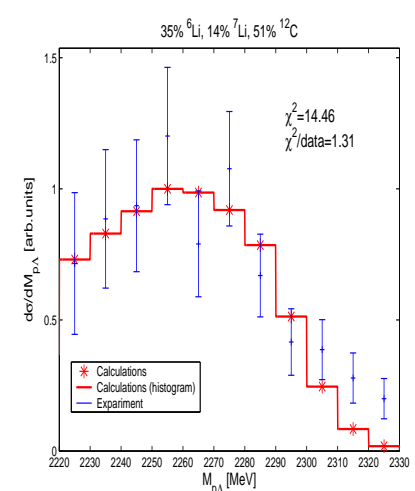

Fig. 4. (Color online) Invariant mass of $\Lambda p$ distribution for $\mathrm{K}^{-}$ absorption in light nuclei in the following proportion: $51 \%{ }^{12} \mathrm{C}$, $35 \%{ }^{6} \mathrm{Li}$ and $14 \%{ }^{7} \mathrm{Li}$. Stars and histogram show our results, while experimental points and error bars are taken from

\section{Concluding remarks}

In summary, we have seen how the experimental $\Lambda p$ invariant mass spectrum of the FINUDA collaboration 25. is naturally explained in our Monte Carlo simulation as a consequence of final state interactions of the particles produced in nuclear $K^{-}$absorption as they leave the nucleus, without the need of resorting to exotic mechanisms like the formation of a $K^{-} p p$ bound state. Together with the interpretation of the peak seen in the proton momentum spectrum [21,24] as coming from a two-nucleon kaon absorption mechanism, where the rest of the nucleus acts as an spectator and the daughter nucleus remains in its ground state given in Refs. 22,24, it seems then clear that there is at present no experimental evidence for the existence of deeply bound kaonic states.

From the theoretical side, the absence of deeply bound kaonic states is supported by sophisticated many-body calculations of the optical potential in dense matter [13, 14,15. A major step forward has been achieved by recent few body calculations, either solving Faddeev equations [35] or applying variational techniques 36, using realistic $\bar{K} N$ interactions and short-range nuclear correlations. These works predict few-nucleon kaonic states bound by 50-70 MeV but having large widths of the order of 100 $\mathrm{MeV}$, thereby disclaiming the findings of Refs. [18, 19,20].

\section{Acknowledgments}

This work is partly supported by contracts BFM200300856 and FIS2005-03142 from MEC (Spain) and FEDER, the Generalitat de Catalunya contract 2005SGR-00343, and the E.U. FLAVIANET network contract MRTN-CT2006-035482. This research is part of the EU Integrated Infrastructure Initiative Hadron Physics Project under contract number RII3-CT-2004-506078.

\section{References}

1. D.B. Kaplan, and A.E. Nelson, Phys. Lett. B175 (1986) 57; 179 (1986) 409(E).

2. E. Friedman, A. Gal, and C.J. Batty, Nucl. Phys. A579 (1994) 518.

3. N. Kaiser, P. B. Siegel and W. Weise, Nucl. Phys. A 594 (1995) 325.

4. E. Oset and A. Ramos, Nucl. Phys. A 635 (1998) 99.

5. J. A. Oller and U. G. Meissner, Phys. Lett. B 500 (2001) 263.

6. M.F.M. Lutz and E.M. Kolomeitsev, Nucl. Phys. A 700, (2002) 193.

7. C. Garcia-Recio, J. Nieves, E. Ruiz Arriola, and M. J. Vicente Vacas, Phys. Rev. D67 (2003) 076009.

8. D. Jido, J. A. Oller, E. Oset, A. Ramos and U. G. Meissner, Nucl. Phys. A 725 (2003) 181.

9. B. Borasoy, R. Nissler and W. Weise, Eur. Phys. J. A 25 (2005) 79 .

10. J. A. Oller, J. Prades and M. Verbeni, Phys. Rev. Lett. 95 (2005) 172502.

11. V. Koch, Phys. Lett. B 337 (1994) 7.

12. M. Lutz, Phys. Lett. B 426 (1998) 12.

13. A. Ramos and E. Oset, Nucl. Phys. A 671 (2000) 481.

14. J. Schaffner-Bielich, V. Koch and M. Effenberger, Nucl. Phys. A 669 (2000) 153

15. A. Cieply, E. Friedman, A. Gal and J. Mares, Nucl. Phys. A 696 (2001) 173

16. S. Hirenzaki, Y. Okumura, H. Toki, E. Oset and A. Ramos, Phys. Rev. C 61 (2000) 055205.

17. A. Baca, C. Garcia-Recio and J. Nieves, Nucl. Phys. A 673 (2000) 335.

18. Y. Akaishi and T. Yamazaki, Phys. Rev. C 65 (2002) 044005;

19. A. Dote, H. Horiuchi, Y. Akaishi and T. Yamazaki, Phys. Rev. C 70 (2004) 044313.

20. Y. Akaishi, A. Dote and T. Yamazaki, Phys. Lett. B 613 (2005) 140.

21. T. Suzuki et al., Phys. Lett. B 597 (2004) 263.

22. E. Oset and H. Toki, Phys. Rev. C 74 (2006) 015207.

23. M. Iwasaki, these Proceedings.

24. M. Agnello et al. [FINUDA Collaboration], Nucl. Phys. A 775 (2006) 35.

25. M. Agnello et al. [FINUDA Collaboration], Phys. Rev. Lett. 94 (2005) 212303.

26. V. K. Magas, E. Oset, A. Ramos and H. Toki, Phys. Rev. C 74 (2006) 025206; Ibid., nucl-th/0611098

27. R.H. Dalitz, T.C. Wong and G. Rajasekaran, Phys. Rev. 153 (1967) 1617

28. A. D. Martin, Nucl. Phys. B 179 (1981) 33.

29. M. Iwasaki et al., Phys. Rev. Lett. 78 (1997) 3067.

30. G. Beer et al. [DEAR Collaboration], Phys. Rev. Lett. 94 (2005) 212302.

31. S. Prakhov et al. [Crystall Ball Collaboration], Phys. Rev. C 70 (2004) 034605.

32. V. K. Magas, E. Oset and A. Ramos, Phys. Rev. Lett. 95 (2005) 052301; E. Oset, V. K. Magas and A. Ramos, AIP Conf. Proc. 842 (2006) 455, hep-ph/0512361 Ibid., 814 (2006) 273, nucl-th/0512090. V. K. Magas, hep-ph/0510122

33. L. Tolos, A. Ramos and E. Oset, Phys. Rev. C 74 (2006) 015203

34. W. Weise, these Proceedings, nucl-th/0701035 
35. N. V. Shevchenko, A. Gal and J. Mares, Phys. Rev. Lett, in print, nucl-th/0610022.

36. A. Doté and W. Weise, these Proceedings, nucl-th/0701050.

37. P. A. Katz, K. Bunnell, M. Derrick, T. Fields, L. G. Hyman and G. Keyes, Phys. Rev. D 1 (1970) 1267.

38. E. Oset, V. K. Magas, E. Oset and H. Toki, these Proceedings, nucl-th/0701023.

39. J. Mares, E. Friedman and A. Gal, Nucl. Phys. A 770 (2006) 84. 